\title{
émulations
}

\section{La construction d'une parenté à la marge}

\section{Les bâtards nobles chez les Bourbon à la fin du Moyen Âge}

Marie-Lise Fieyre

Émulations - Revue de sciences sociales 2019, n 32, «Aux frontières de la parenté. Un éclairage par les marges ».

Article disponible à l'adresse suivante

https://ojs.uclouvain.be/index.php/emulations/article/view/fieyre

\section{Pour citer cet article}

Marie-Lise Fieyre, « La construction d'une parenté à la marge. Les bâtards nobles chez les Bourbon à la fin du Moyen Âge », Émulations, n 32, Mise en ligne le 20 avril 2020. DOI : 10.14428/emulations.032.03

Distribution électronique : Université catholique de Louvain (Belgique) : ojs.uclouvain.be

(C) Cet article est mis à disposition selon les termes de la Licence Creative Commons Attribution, Pas d'Utilisation Commerciale 4.0 International. http://creativecommons.org/licenses/by-nc/4.0/

Éditeur : Émulations - Revue de sciences sociales / Presses universitaires de Louvain https://ojs.uclouvain.be/index.php/emulations

ISSN électronique : 1784-5734

$\frac{\text { PUL PRESSES }}{\text { UNIVERSITAIRES }}$ 


\title{
La construction d'une parenté à la marge
}

\author{
Les bâtards nobles chez les Bourbon \\ à la fin du Moyen Âge
}

Marie-Lise Fieyre ${ }^{1}$

\begin{abstract}
[Résumé] Symboles de la transgression du mariage chrétien, les enfants naturels sont frappés d'incapacités juridiques, définies par le droit coutumier, l'Église et la jurisprudence à partir du Xle siècle. L'objectif est de préserver les intérêts successoraux des enfants légitimes. Ceux qui naissent hors mariage se trouvent distingués au sein de la parenté. À travers la famille ducale de Bourbon (XIVe-XVle siècle), l'objectif est de comprendre la place donnée aux enfants naturels nobles au sein d'un groupe de parenté. Si leur exclusion successorale n'entraîne pas une exclusion sociale, ils restent volontairement positionnés à la marge de la parenté et destinés à servir les intérêts du lignage. Mots-clés : Moyen Âge, bâtardise, Bourbon, parenté.
\end{abstract}

The Construction of Kinship at the Margins: Noble Bastards at the Bourbons in the Late Middle Ages

[Abstract] Natural children epitomize the transgression of Christian marriage, and thus are struck with legal inability as defined by customary law, the Church, and jurisprudence from the $11^{\text {th }}$ century onwards. This aimed at preserving the successional rights of legitimate children. Those born outside lawful marriage found themselves discriminated inside a family group. Through the Bourbon ducal family $\left(14^{\text {th }}-16^{\text {th }}\right.$ centuries), the aim is to understand what place was given to natural children inside a family group of the nobility. Even though their successional exclusion did not transpose on a social point of view, they remained and were deliberately kept on the fringe of their family groups and were fated to serve the interests of the lineage.

Keywords: Middle Age, bastardy, Bourbon, kinship, language of identity.

Dans son traité d'héraldique, Bartolo de Sassoferrato († 1357) préconise de différencier les armoiries des enfants naturels en y ajoutant une figure pour les distinguer des autres membres du lignage. Cette pratique se diffuse largement en Europe dans les deux derniers siècles du Moyen Âge (Hablot, 2016 : 258-259). L'héraldique est alors l'une des manières de symboliser la distinction de l'enfant né hors mariage vis-à-vis du groupe de parenté.

À partir du XI ${ }^{\mathrm{e}}$ siècle, l'Église tente d'imposer le mariage comme cadre privilégié de la procréation. Une distinction s'organise progressivement entre les personnes nées en légitime mariage et les autres (Lefebvre-Teillard, 2008: 239-258; Roumy, 2000, 2009). La fin du Moyen Âge connaît pourtant un « essor » des bâtards nobles, particulièrement visible dans les politiques princières (Harsgor, 1975 ; Bousmar et al., 2015).

\footnotetext{
${ }^{1}$ Université de Paris, ICT, F-75013 Paris, France.
} 
Pour aborder ce paradoxe, nous proposons de nous focaliser sur la « maison » ducale de Bourbon, qui appartient à la haute noblesse du royaume de France ${ }^{2}$. Elle constitue un observatoire privilégié car les enfants naturels représentent $28 \%$ du groupe de parenté3. Les sources exploitées proviennent principalement des documents de la pratique, produits par (et sur) les Bourbon, comme les actes notariés, les transactions foncières ou les quittances. Les membres nés hors mariage y sont mentionnés au même titre que ceux de naissance légitime. Leur positionnement dans la parenté apparaît également par des sources iconographiques, comme l'héraldique ou l'emblématique.

Centrer l'étude sur un groupe de parenté limité permet de proposer une approche au plus près des acteurs et de leurs interactions ${ }^{4}$. Cette démarche s'inscrit dans une histoire - développée depuis les années 1980 - qui considère la famille dans toutes ses dimensions (Lett, 2017). Les bâtards constituent aujourd'hui des points d'observation privilégiés pour reconsidérer la parenté et la famille médiévales dans leur complexité et leur plasticités. Ce faisant, nous proposons une relecture des modes de construction de la bâtardise à travers la place spécifique des enfants naturels dans le groupe et dans les rapports de parenté.

Les enfants naturels font d'abord l'objet d'une construction juridique, linguistique et symbolique à laquelle ils participent. Ainsi distingués, ils occupent une place déterminée à la marge du groupe de parenté, et assurent des fonctions ou des rôles parfois fermés aux personnes de naissance légitime.

\section{Des enfants naturels mis à la marge sans être marginalisés}

À partir de 1493, le duc Pierre de Bourbon ordonne l'enregistrement des coutumes de Bourbonnais, publiées cinq ans plus tard. Le neuvième article concerne les «droitz que le seigneur prent sur les bastard $z^{6} »$. Cette coutume exclut les enfants naturels de la succession de leurs père et mère, ou de leurs descendants directs, car le « bastard n’a point de souche ${ }^{7}$ ». Le seigneur haut justicier est également institué héritier si la personne née hors mariage décède sans héritier (Chevailler, 1957) L’enfant naturel paraît ainsi juridiquement isolé du groupe familial. En outre, le dernier item de l'article concerne « tous estrangiers nés hors du royaulme » sur lesquels s'appliquent les mêmes

\footnotetext{
2 Sur la notion de « maison », voir le bilan critique proposé par Elie Haddad (2014).

${ }^{3}$ Le groupe pris en compte comprend 152 personnes dont 43 nées hors mariage.

${ }^{4}$ L'approche pratique de la parenté s'inscrit dans une histoire qui emprunte beaucoup à la sociologie (Weber, 2005).

${ }^{5}$ La parenté est définie comme « l'ensemble des rapports de parenté (par consanguinité, affinité ou parenté spirituelle) constituant un système propre à chaque société » (Morsel, 2017 : 26). Le terme de famille est critiqué, notamment en raison de sa grande polysémie au Moyen Âge (Morsel, 2017 : 80-86). Il peut toutefois être envisagé comme une parentèle - étendue à ceux qui partagent un même toit (familia) - formée par des interactions répétées et constituant une communauté émotionnelle (Lett, 2017).

${ }^{6}$ En ce livre sont les coutumes et statues du païs et duché de Bourbonnois, notairement tenues et gardées en icelluy païs, Lyon, H. Marion, 1498, fo $9 v^{\circ}-10 v^{\circ}$. La lettre de commission du duc (1493) a été éditée par Aurroux des Pommiers (1780), vol. 2 : 20.

7 En ce livre sont les coutumes... op. cit., fo $10 \mathrm{r}^{\circ}$.
} 
prérogatives seigneuriales. Les bâtards sont ainsi rapprochés des aubains, ce qui accentue l'analogie entre les deux ${ }^{8}$. Le droit coutumier n'est pas le seul à proposer un traitement différencié pour les enfants naturels. La doctrine théologique et canonique d'un mariage sacramentel est progressivement constituée entre le $\mathrm{XI}^{\mathrm{e}}$ et le XIII ${ }^{\mathrm{e}}$ siècle (Gaudemet, 1987 :139-149, 188-191). Dès lors, toute personne non issue d'un légitime mariage est juridiquement considérée comme bâtarde?.

Quel que soit le droit sollicité, l'incapacité juridique des enfants naturels implique une mise à l'écart de la parenté, ou d'une partie de celle-ci. Or, leur statut reste juridiquement défini, ce qui leur confère une place spécifique sans être rejetés du corps social (Geremek, 1976:361; Schmitt, 1988:277-280). Les enfants naturels ne se trouvent donc pas hors des normes de la société médiévale, comme le seraient les marginaux. Ils constituent plutôt une part a-normale de cette société en étant définis par leur non-conformité à des préceptes qui agissent comme des " opérateurs discriminants » (Boisselier, $2010: 27-33)^{10}$.

Notons également que les sources juridiques ne possèdent pas seulement une fonction normative. En élaborant des hiérarchies, elles reflètent une manière de concevoir le monde et promeuvent un idéal d'organisation sociale. Les phénomènes de marginalité y apparaissent prioritairement comme une infraction aux normes. Au contraire, les documents de la pratique permettent de considérer les agissements des acteurs en fonction de systèmes de valeurs et d'intérêts propres (Boisselier, 2010 : 17-19). Ils montrent également que l'usage judiciaire des normes matrimoniales a fortement contribué à l'éviction des enfants naturels vis-à-vis de l'héritage parental (McDougall, 2017). Ainsi, à la fin du Moyen Âge, le principe d'exclusion successorale des enfants naturels est strictement appliqué chez les Bourbon, même si certains illégitimes sont mentionnés dans des testaments pour des legs mineurs. La famille renforce même le dispositif juridique en préférant les collatéraux légitimes aux bâtards directs. En 1488, le duc Jean II de Bourbon meurt sans descendance légitime. Il est toutefois le père de nombreux enfants naturels, dont quatre fils sont encore en vie. L'aîné, Mathieu, demeuré laïc, ne réclame jamais le titre ducal, qui fait l'objet d'un accord entre les deux frères légitimes survivants du duc ${ }^{11}$. Son éviction de l'héritage élimine toute revendication successorale. La personne née hors mariage est ainsi mise à la marge d'une parenté considérée comme

\footnotetext{
8 /bid., fo $10 r^{\circ}-10 v^{\circ}$. Ces dispositions se retrouvent dans d'autres coutumes (Chevailler, 1957, 1958).

${ }^{9}$ Terme générique employé dans les documents de la pratique à la fin du Moyen Âge. Le vocabulaire juridique varie selon la typologie des filiations (Roumy, 2009 : 283-284).

${ }^{10}$ Expression employée par Stéphane Boisselier (2010 : 27) comme une différentiation entraînant un traitement séparé entre plusieurs groupes distincts. La multiplication des typologies de filiation à partir de la seconde moitié du XII siècle " n'a pas pour but d'introduire de nouvelles "discriminations" » mais de faire entrer "le plus d'enfants possible dans la catégorie des légitimes et des légitimables » (Roumy, 2009 : 286).

${ }^{11}$ Archives nationales de France (AN), P 1373', n²138, éd. La Mure (1982), III : 211-213, preuve $129 e$.
} 
le groupe des personnes susceptibles de transmettre le patrimoine : aînés puis cadets masculins et féminins ${ }^{12}$.

La notion de marge se révèle alors particulièrement pertinente pour les enfants naturels, si elle est envisagée comme un interstice, une zone limite, voire une variable d'ajustement de la parenté. Elle naît de « l'expression d'une tension, entre appartenance et exclusion, inhérente au système qui la produit, de relations hiérarchisées, de rapports de domination » (Le Dantec-Mowry et al., 2018:17). Les bâtards sont associés et dépendants de la parenté légitime tout en étant différents. Ils ne sont ni marginaux, ni marginalisés, mais ils se positionnent in margine : sur (ou à) la marge, pour reprendre un vocable médiéval ${ }^{13}$.

Considérer les enfants naturels comme un groupe à la marge de la parenté légitime implique également d'observer la (ou les) zone(s) de contact(s) entre la parenté naturelle et la parenté légitime. Les droits et leurs usages constituent une frontière normative. Les juristes médiévaux définissent les enfants naturels à partir des éventuels empêchements matrimoniaux touchant leurs géniteurs. Ils limitent ensuite l'accès à une légitimation en fonction du statut juridique de naissance des personnes (LefebvreTeillard, 2008 : 239-273 ; Roumy, 2009). Les gradations d'illégitimité qui en résultent sont complexifiées par les recours eux-mêmes. En effet, l'Église établit des formes de légitimation par rescrit et des dispenses apostoliques pour défaut de naissance qui atténuent ou effacent la macula ${ }^{14}$. Les usages qui en sont faits s'ajoutent aux subtilités juridiques. Chez les Bourbon, les légitimations sont extrêmement limitées. Pour Mathieu - cité précédemment - seule une légitimation comportant une clause successorale lui aurait permis de revendiquer l'héritage ducal en 1488. Or, le bâtard n'est - volontairement - jamais légitimé. Même sa reconnaissance officielle tardive par le duc Jean II semble avoir été sujette à débat ${ }^{15}$. Les Bourbon sollicitent des lettres de légitimation (par rescrit du prince ou du pape) si elles ne portent pas atteinte aux intérêts des autres membres légitimes du groupe. De tels choix témoignent à la fois d'un usage très mesuré des opportunités juridiques de légitimation et d'une gestion de la frontière normative séparant enfants naturels et légitimes au sein de la parenté.

Ainsi, les enfants naturels constituent une minorité au sein de la parentèle et à la marge de la parenté légitime. Ils s'inscrivent dans un contexte médiéval très hiérarchisé qui relègue les non-conformes, tout en appliquant une tolérance étendue pour éviter

\footnotetext{
${ }^{12}$ Dans la pratique, les fils naturels concurrencent parfois les filles légitimes (Steinberg, 2012).

${ }^{13}$ Voir les travaux proposés sur le concept de marge, notamment en géographie (Prost, 2004). Les termes employés ici sont généralement exogènes au monde médiéval (Boisselier, 2010 : 21-24), ce qui pose problème pour désigner les catégories sociales de cette période (Aurell, 2005). L'idée de marge est essentiellement employée pour qualifier les bordures des manuscrits ou un espace géographique spécifique (Jacquart, Burnett, 2005 ; Schmitt, 1988 : 282).

${ }^{14}$ La dispense permet à un clerc de naissance illégitime d'accéder aux ordres majeurs. Elle n'efface pas l'illégitimité et n'a aucune influence sur le temporel qui relèvent de la légitimation par rescrit du pape, ou du prince à partir du XIIIe siècle (Lefebvre-Teillard, 2008 : 171-184, 315-327 et 329-341). ${ }^{15}$ Jean Berry, secrétaire du duc Jean II, aurait « retardé ou empesché ledict adveu » : Bibliothèque nationale de France (BnF), ms. fr. 4647, fo $119 v^{\circ}$.
} 
les exclusions sociales ${ }^{16}$. Néanmoins, la bâtardise est également construite par des interactions sociales qui vont au-delà de la norme et qui participent à l'identification du groupe «bâtard».

\section{La construction d'une frontière sociale entre enfants naturels et légitimes}

À la fin du Moyen Âge, d'autres procédés viennent renforcer la distinction sociale des enfants naturels. Il s'agit notamment des signes d'identification, comme l'héraldique et les titulatures, qui sont assimilés et exploités par les lignages nobles. Parmi les Bourbon, Mathieu - précédemment évoqué - reprend les armoiries de son père en les limitant à une pièce. Il porte ainsi d'argent à la bande de Bourbon. La pièce armée sur champ plain est un mode de brisure traditionnellement employé par les enfants naturels de la famille ${ }^{17}$. Par ailleurs, sa titulature fait état de son statut en associant le titre de « bâtard de Bourbon » à son nomen ${ }^{18}$. L'illégitimité de naissance apparaît donc dans des signes identifiants différents : à travers un figuré ou des termes spécifiques qui la mentionnent explicitement. Dans les deux cas, elle constitue un élément fondamental de l'identité de la personne durant la période considérée.

À en croire les traités d'héraldique, la distinction sert à isoler les bâtards des membres légitimes du groupe, afin de préserver celui-ci. En fait, les brisures héraldiques mettent surtout en lumière des liens de parenté. Elles se développent dans le courant du XIII ${ }^{e}$ siècle et celles spécifiques aux illégitimes commencent à se mettre en place à partir du siècle suivant (Hablot, 2016 : 258-260 ; Loutsch, 1988 : 155-157). L'expression «N. bâtard de » se structure aussi progressivement dans les titulatures nobles à partir du XIVe siècle (Fieyre, 2017 : 102-104). La fin du Moyen Âge est donc une époque où les bâtards (et les cadets légitimes) gagnent en visibilité publique. Le statut juridique de naissance pour les enfants naturels mais aussi le rang dans la fratrie ou le sexe pour les personnes nées en légitime mariage deviennent des pivots des constructions identitaires. La volonté est moins de stigmatiser que de faire valoir l'organisation des rapports de parenté. Les hiérarchies internes apparaissent en fonction de la capacité (ou de l'incapacité) de chacun d'accéder à l'héritage. Par ces pratiques, les personnes concernées revendiquent aussi une filiation conforme à leurs intérêts. Les cadets de Bourbon empruntent parfois des signes héraldiques ou emblématiques aux armoiries maternelles. Ces reprises sont toujours motivées par des raisons successorales ou

\footnotetext{
${ }^{16}$ Nous reprenons ici un concept définit par Stéphane Boisselier (2010) et remis en question en conclusion du collectif par Martin Aurell (2010).

${ }_{17}$ Visibles sur son sceau : BnF, Clairambault 122, n² 27 (1492). L'argent est le seul émail employé pour le champ plain par les bâtards de Bourbon : BnF., Estampes Rés. Oa 12, fo 8 ; BnF., ms. fr. $1989, f^{\circ} 146 r^{\circ}$ et $154 r^{\circ}$. En héraldique, le champ correspond à la surface de l'écu. La pièce est une figure géométrique posée sur le champ qui reste visible. Le champ est qualifié de « plain » lorsqu'il est d'un seul émail, sans figures.

${ }^{18} \mathrm{AN}, \mathrm{P} 1397^{3}, \mathrm{n}^{\circ} 625$.
} 
politiques. N'étant pas concernés, les bâtards réservent l'exclusivité de leurs emprunts au lignage paternel, pour bénéficier de son capital social, économique ou symbolique.

Les enfants naturels ont donc accès à certaines formes de succession ${ }^{19}$. D'un côté, ils partagent une partie du capital symbolique du lignage (nom, armoiries), qui les associe au groupe de parenté. D’un autre côté, ils demeurent explicitement évincés au profit des héritiers éventuels (les enfants légitimes : hommes ou femmes). La frontière normative différenciant enfants naturels et légitimes est donc renforcée par une frontière sociale (Lamont, Molnár, 2002 : 168). La distinction des bâtards au sein de la parenté crée une limite interne, objectivée par des marqueurs d'identification, qui accompagnent les spécificités liées à la bâtardise ${ }^{20}$. Les personnes nées hors mariage restent incapables de jouir de certaines prérogatives (successorales ou symboliques) réservées aux enfants légitimes ce qui limite leurs opportunités sociales. Les allusions constantes à la parenté par les marqueurs d'identification prouvent également que la frontière n'est pas une fracture, car les relations sont maintenues. Les enfants naturels font donc partie intégrante du groupe de parenté.

Les contours identitaires du groupe constitué restent toutefois incertains. Chez les Bourbon, les conditions juridiques exactes de la naissance (enfant naturel simple, adultérin, incestueux) importent peu sur le devenir de la personne et n'ont aucune incidence sur la manière de dire l'illégitimité. En revanche, une dispense ou une légitimation sont susceptibles d'entraîner la modification de certains signes identifiants. En 1470, la titulature de l'évêque du Puy « Jehan de Bourbon » ne fait pas mention de son illégitimité de naissance, à l'instar de tous les autres illégitimes entrés dans les ordres ${ }^{21}$. Il conserve toutefois une brisure sur ses armoiries, comme d'autres membres du clergé nés hors mariage. Treize ans plus tard, Louis, fils naturel du duc Charles $I^{\text {er }}$ de Bourbon, est légitimé par le roi Louis XI. Sa titulature se modifie à la même période, en passant de « Loys bastard de Bourbon » à « Loys de Bourbon ». En parallèle, il conserve, lui aussi, la brisure sur ses armoiries ${ }^{22}$. Sous certaines conditions, la marque de l'illégitimité peut donc être retirée de certains éléments d’identification, alors qu'elle est maintenue sur d'autres.

Ces variations entre les marqueurs de l'illégitimité ont également des répercussions sur la population légitime. La titulature est attachée à la personne et disparaît avec elle. En revanche, les armoiries (brisées) se transmettent aux descendants éventuels. Ainsi, les seigneurs de Lavedan héritent des armes de Charles, bâtard de Bourbon († 1502) jusqu'au XVII siècle (Pinoteau, 1979 : 40-41). Des individus nés en légitime mariage peuvent donc être amenés à porter les signes de l'illégitimité d'un aïeul. L’ensemble de ces variations dans l'affirmation identitaire d'une naissance hors mariage ne modifie

\footnotetext{
${ }^{19}$ La succession comprend la dévolution d'un capital symbolique quand l'héritage concerne seulement le patrimoine et les biens matériels (Derouet, Goy, 1998 : 135).

${ }^{20}$ Le langage (scripturaire ou figuré) possède une fonction performative dans la construction des frontières et des catégories. Les frontières sont donc à la fois le produit d'actions et d'interactions (Staszak, $2017: 11$ ).

21 BnF, Clairambault 952, n 97, du 20 juin 1470.

${ }^{22} \mathrm{BnF}$, ms. fr. 2909, fo 47, du 10 octobre 1482 ; BnF, ms. fr. 20390, n 51, du 29 août 1483.
} 
en rien le statut juridique des personnes concernées. En revanche, elles brouillent la perception publique de la frontière sociale entre bâtard et enfant légitime. Si l'on considère que le langage (scripturaire ou visuel) possède une fonction performative, c'est la définition même de la bâtardise (comme conséquence sociale d'une illégitimité de naissance) qui est affectée. D’une part, les contours de l'identité bâtarde sont flous car ils peuvent englober des personnes de naissance légitime. D’autre part, les gradients juridiques et sociaux observés à cette période créent des formes de bâtardise qui complexifient le groupe formé par la population née hors mariage. Les dispenses et les légitimations finissent de perméabiliser les contours du groupe.

La limite distinguant les enfants naturels au sein de la parenté est donc floue. Elle résulte d'abord de l'enchevêtrement des statuts des intéressés : naturels ou légitimes, clercs ou laïcs, liés au statut d'un ascendant, ce qui souligne aussi l'hétérogénéité du groupe formé par les bâtards. Certaines caractéristiques sont ensuite partagées au sein de la parenté, comme le sexe ou la noblesse. Enfin, des opportunités liées à une dispense ou une légitimation entraînent des variations dans l'emploi des marqueurs de l'illégitimité. Malgré ces nuances et l'hétérogénéité du groupe, la distinction des enfants naturels au sein de la parenté reste confortée par le lignage qui y trouve un intérêt.

\section{Une différentiation utile au lignage}

La position des personnes nées hors mariage n'est pas seulement organisée par un cadre normatif et culturel propre à l'Occident tardo-médiéval. Dans les lignages nobles, comme celui des Bourbon, elle révèle surtout des hiérarchies familiales exploitées à des fins politiques.

Ces hiérarchies apparaissent notamment à travers les possessions foncières des individus et leurs modes de circulation. En 1390, Jean de Bourbon et son épouse, Catherine de Vendôme, répartissent leurs biens entre leurs enfants légitimes ${ }^{23}$. Les domaines féodaux les plus prestigieux sont attribués aux enfants légitimes par ordre de naissance et en fonction des biens disponibles. Les bâtards, eux, ne peuvent pas prétendre aux terres les plus importantes. En outre, les biens fonciers qu'ils reçoivent proviennent majoritairement de donations entre vifs, dans le cadre d'une parenté proche. En 1486 le duc Jean II de Bourbon fait une série de donations à deux de ses fils bâtards. Il remet le château de Bouthéon puis la baronnie de Roche-en-Régnier à son aîné, Mathieu²4. En parallèle, son cadet obtient les terres et seigneuries de Lachaussée, Étain et Bouconville ${ }^{25}$. Le choix de ces terres révèle à la fois la position sociale des enfants naturels dans la hiérarchie familiale et leur fonction au sein du lignage.

\footnotetext{
${ }^{23}$ AN, P $1362^{2}, n^{\circ} 1221$. Carency, Pas-de-Calais, arr. Lens, con Bully-les-Mines.

${ }^{24} \mathrm{AN}, \mathrm{P} 1397^{3}, \mathrm{n}^{\circ} 621^{1 \text { et } 2}$; AN, P 1397³, $\mathrm{n}^{\circ} 624$ et 625. Bouthéon (Andrézieux-Bouthéon), Loire, arr. Montbrison, $c^{\text {on }}$ Saint-Galmier ; Roche-en-Régnier, Haute-Loire, arr. Le Puy-en-Velay, con du plateau du Haut-Velay granitique.

${ }^{25}$ BnF, Clairambault 640, fo 74-75. Lachaussée, Meuse, arr. Commercy, con Saint-Mihiel ; Étain, Meuse, arr. Verdun, ch. I. con ; Bouconville, Ardennes, arr. Vouziers, con Attigny.
} 
Ce sont d'abord des biens fonciers mineurs, par rapport au reste du patrimoine familial. Chez les Bourbon, les enfants nés hors mariage se retrouvent à la tête de seigneurie ou de baronnies, alors que les cadets peuvent prétendre à diriger des comtés. Les rares titres comtaux détenus par des bâtards de Bourbon sont reçus par mariage ou par donation royale ${ }^{26}$. Ensuite, les biens concédés par Jean II à ses fils naturels sont tous des acquêts récents. Les terres de Lachaussée, Étain et Bouconville appartenaient à René d'Anjou qui en a fait don au duc de Bourbonnais en $1478^{27}$. Jean II achète également le château de Bouthéon (en Forez) en 1462 et la baronnie de Roche-en-Régnier en $1481^{28}$. L'ensemble des territoires est donc en possession des Bourbon depuis moins de trois décennies. Enfin, ces biens fonciers cédés aux bâtards sont excentrés par rapport aux territoires ducaux. Seul le château de Bouthéon se trouve en Forez qui avait été définitivement annexé à la principauté en 1382 (Mattéoni, 1998 : 75-79). La seigneurie de Roche-en-Régnier comprend également les terres d'Artias, de Retournac, d'Espalion et de Malivernas qui suivent le cours de la Loire ${ }^{29}$. Elles se trouvent toutes au-delà de la frontière sud de la principauté. De leur côté, les terres jadis possédées par René d'Anjou se situent à la frontière nord-est du royaume, dans le duché de Bar, très loin des possessions bourbonnaises ${ }^{30}$.

Les distinctions juridiques et identitaires des bâtards ont des conséquences sociales et spatiales. Elles s'expriment d'abord par une relégation en matière de capital symbolique, avec des terres et des titres moins prestigieux que ceux dévolus aux cadets. Elles se confirment ensuite par une inégalité d'accès au patrimoine familial, en leur conférant seulement des acquêts. Elle s'incarne enfin par une mise à la frontière (géopolitique) des enfants naturels. L'ensemble de ces dispositions montre la nécessité de maintenir les bâtards dans une situation d'infériorité par rapport aux cadets légitimes. L'enfant né hors mariage ne doit jamais remettre en cause les intérêts des autres membres du groupe de parenté. Une déchéance sociale complète lui est toutefois évitée pour deux raisons principales. Tous les enfants, sans distinction de naissance, sont protégés par le droit aux aliments. Les parents doivent subvenir à leurs besoins (nourriture, éducation) jusqu'à leur autonomie financière. Le soutien doit également être conforme au statut social du (ou des) géniteur(s) (Lefebvre-Teillard, 1976 : 264-266). À cette protection juridique s'ajoute l'intérêt des lignages nobles. Les enfants naturels doivent leur statut social au groupe de parenté qui les reconnaît et constituent souvent des alliés dévoués

\footnotetext{
${ }^{26}$ En 1481, le roi Louis XI a ainsi donné à Louis, fils naturel du duc Charles ler de Bourbon, le comté de Ligny confisqué sur les biens du connétable de Saint-Pol : AN, X1A 8608, f 4 et 21 vº. Ligny-enBarrois, Meuse, arr. Bar-le-Duc, ch. I. con.

${ }^{27}$ En restitution d'une partie de la dot de Marie de Bourbon, duchesse de Calabre († 1448) : AN, $\mathrm{P} 1359^{2}, \mathrm{n}^{\circ} 737$ et $\mathrm{AN}, \mathrm{P} 1379^{1}, \mathrm{n}^{\circ} 3105$.

${ }^{28}$ AN, P 1359², n 765 et Mathevot C. (2011 : 118-119). Sur la baronne de Roche-en-Régnier : La Mure J.-M. (1982, II : 469).

${ }^{29}$ Le château d'Artias se situe sur l'actuelle commune de Retournac, Haute-Loire, arr. Yssingeaux, $c^{\text {on }}$ Bas-en-Basset ; Espalion, Haute-Loire, arr. Le Puy-en-Velay, con Emblavez-et-Meygal, c. Vorey ; Malivernas, Haute-Loire, arr. du Puy-en-Velay, con Plateau du Haut-Velay granitique, c. Saint-Pierre du Champ.

${ }^{30}$ Bar-le-Duc, Meuse, ch. I. arr.
} 
au chef de famille (Fieyre, 2017 : 574-578 ; Schnerb, 2015). Ils représentent alors des acteurs de choix pour maîtriser des territoires frontaliers, voire éloignés, où la légitimité politique du prince peut être contestée. Dans un lignage noble, le bâtard doit donc avoir un rang social inférieur aux cadets mais suffisant pour soutenir la politique princière en possédant ce type de biens féodaux.

L'exploitation de l'infériorité sociale des enfants naturels se retrouve également dans les conséquences sociales des unions matrimoniales, masculines et féminines. Les filles du duc Charles Ier de Bourbon se marient avec des dots numéraires très différentes. L'aînée, Marie, apporte 150000 écus d'or à son époux, Jean de Calabre ${ }^{31}$. Marguerite, sa cadette, n'obtient que 60000 écus d'or lors de son union avec Philippe de Savoie ${ }^{32}$. Les montants alloués aux filles légitimes restent néanmoins très supérieurs à ceux destinés aux bâtardes. Sidoine, sœur consanguine des précédentes, se marie avec une dot de 1000 écus d'or ${ }^{33}$. La dot est l'un des moyens permettant d'appréhender les variations sociales et prouve la différentiation socio-économique des enfants naturels. Elle est aussi proportionnelle au rang de l'époux. Là où les filles légitimes épousent des héritiers de duchés ou de comtés, les filles naturelles s'unissent à des officiers. Ces derniers appartiennent à une noblesse moins prestigieuse, ce qui les rend inaccessibles aux enfants légitimes de Bourbon. Ils sont aussi le plus souvent inféodés au prince. Les filles naturelles présentent donc l'opportunité de renforcer les liens existants entre le chef de lignage et ses propres vassaux (Fieyre, 2017 : 245-256). Pour affirmer son pouvoir, le duc de Bourbonnais s'appuie, en effet, principalement sur la noblesse régionale (Mattéoni, 2009). Les unions contractées par les enfants naturels possèdent donc un intérêt politique et stratégique majeur, dans un contexte marqué par une transformation des relations féodo-vassaliques avec une contractualisation progressive des liens et l'affirmation du pouvoir royal.

\section{Conclusion}

Dans la seconde moitié du XVIe siècle, Jean Bacquet définit le bâtard comme une personne n'ayant « ne race, ne gent, ne ligne », à l'instar de certaines coutumes du siècle précédent ${ }^{34}$. Cette acception - purement juridique - témoigne d'une mise à distance théorique des enfants naturels : ils représentent les « sauvageons » de l'arbre symbolisant la lignée de laquelle ils sont issus (Steinberg, 2016 : 270-280).

La distinction des personnes nées hors mariage transparaît également dans les discours de la fin du Moyen Âge. Elle est le fruit d'une construction progressive, effectuée sur plusieurs siècles. L'éviction des illégitimes est d'abord juridique et religieuse. L'Église forge une norme matrimoniale qui établit une hiérarchie et qui valorise les

\footnotetext{
${ }^{31} \mathrm{AN}, \mathrm{P} 1370^{2}, \mathrm{n}^{\circ} 1915^{1}$.

32 lbid., n 1928.

33 BnF, ms. fr. 20178, fo 121-123.

${ }^{34}$ Bacquet J. (1688), Second traité des droits du domaine de la couronne de France concernant le droit de bâtardise, dans Les œuvres de Me Jean Bacquet, avocat du roy en la chambre du Tresor, augmentées de plusieurs questions, décisions et arrests des cours souveraines de France, par Maistre Claude de Ferrière, Paris.
} 
personnes nées d'un légitime mariage au détriment de celles venues au monde en dehors de ce cadre. La norme juridique et les usages qui en sont faits renvoient progressivement les bâtards aux marges de la parenté.

Cette distinction gagne en visibilité à partir du XIV siècle, lorsqu'elle imprègne les langages identitaires, scripturaires ou figurés. Le statut juridique de naissance devient alors une composante essentielle de l'identité publique, notamment dans la noblesse. La mention de l'illégitimité dans les sources témoigne d'une meilleure visibilité sociale des enfants naturels. Elle permet également aux historiens de les identifier plus aisément. Par extension, elle renforce la distinction faite entre les personnes nées dans, et hors, d'un mariage valide, ce qui fixe plus précisément la place de chacun par rapport à l'héritage. Les aînés légitimes (masculins) forment le noyau dur de la parenté : celui sur lequel repose la transmission principale du patrimoine et le maintien du rang social du groupe. Autour de lui gravitent les membres qui peuvent prétendre à une part de l'héritage : les cadets et les femmes.

L'exemple des Bourbon montre que la distinction juridique et identitaire des enfants naturels leur permet de former un groupe de parents à la marge du noyau lignager légitime, sans exclusion sociale et sans faire d'eux des marginaux au sein de la société. Les bâtards renforcent ainsi le lignage sans remettre en cause les fondements de sa perpétuation. Les normes et les usages sont assimilés par les acteurs (quel que soit leur statut juridique de naissance) qui agissent en fonction de leurs intérêts. Les pratiques qui en découlent ont tendance à renforcer la construction du groupe de parents illégitimes aux marges du lignage, mais sans occulter son hétérogénéité ou les opportunités de quitter le statut de bâtard.

Les Bourbon, comme d'autres lignages nobles de la même époque, offrent ainsi l'exemple d'un groupe de parenté qui organise et utilise la minorité constituée par ses membres naturels. Les illégitimes disposent d'un statut spécifique qui leur confère souvent des rôles ne pouvant pas être tenus par les cadets. Ils permettent aux Bourbon d'étendre leur influence politique par de nouveaux réseaux matrimoniaux et de servir leurs intérêts géopolitiques par la possession de territoires stratégiques. À la fin du Moyen Âge, les bâtards nobles sont donc bien inclus dans une parentèle, dotés d'un statut spécifique et destinés à renforcer la puissance des lignages. Dans le royaume de France, ce système se transforme à partir du milieu du XVI siècle. Les bâtards nobles voient alors leur statut social progressivement remis en cause (Avignon, 2016 : 18-20 ; Steinberg, $2016: 283$ sq.).

\section{Bibliographie}

Aurell M. (2005), « Complexité sociale et simplification rationnelle: dire la stratification au Moyen Âge », Cahiers de civilisation médiévale, vol. 48, fasc. 189, p. 5-15.

Aurell M. (2010), « La remise en question d'un concept », in S. Boisselier, F. ClÉment, J. Tolan (dir.), Minorités et régulations sociales en Méditerranée médiévale, Rennes, PUR, p. 333-343. 
Aurroux des Pommiers M. (1780), Coutumes générales et locales du païs et duché de Bourbonnois, 2 vol., Riom.

Boisselier S. (2010), « De la différenciation sociale à la minoration en passant par les régulations, quelques propositions », in S. Boisselier, F. Clément, J. Tolan (dir.), Minorités et régulations sociales en Méditerranée médiévale, Rennes, PUR, p. 15-48.

Bousmar É., Marchandisse A., Masson C., Schnerb B. (dir.) (2015), La bâtardise et l'exercice du pouvoir en Europe du XIII a a début du XVI ${ }^{e}$ siècle, Lilles, Revue du Nord, Hors-Série, $\mathrm{n}^{\circ}$ 31, coll. « Histoire».

Chevailler L. (1957), «Observations sur le droit de bâtardise dans la France coutumière du XII ${ }^{\mathrm{e}}$ au XVI ${ }^{\mathrm{e}}$ siècle », Revue historique de droit français et étranger, p. 376-411.

Chevailler L. (1958), « Notes sur le testament du bâtard dans les coutumes du Nord», Revue du Nord, $\mathrm{n}^{\circ}$ 40, p. 207-208.

Derouet B., Goy J. (1998), « Transmettre la terre. Les inflexions d'une problématique de la différence », in G. Bouchard, J. Goy, A.-L. Head-König, Problèmes de la transmission des exploitations agricoles (XVIII -XX ${ }^{e}$ siècle), Mélanges de l'École française de Rome. Italie et Méditerranée, Rome, vol.110, n¹, p.117-153.

FIEYRE M.-L. (2017), Bâtards de princes. Identité, parenté et pouvoir des enfants naturels chez les Bourbon (XIV'-milieu du XVI siècle), thèse de doctorat, Paris, Université Sorbonne Paris-Cité / Université Paris Diderot - Paris 7.

Gaudemet J. (1987), Le mariage en Occident. Les mœurs et le droit, Paris, Édition du Cerf.

GEREMEK B. (1976), Les marginaux parisiens aux XIVe et XVe siècles, Paris, Flammarion.

HaвLот L. (2016), « L'héraldique au service de l'histoire. Les armoiries des bâtards à la fin du Moyen Âge, études de cas ", in C. Avignon, Bâtards et bâtardises dans l'Europe médiévale et moderne, Rennes, PUR, p. 257-277.

HADDAD E. (2014), «Qu'est-ce qu'une “maison” ? De Lévi-Strauss aux recherches anthropologiques et historiques récentes », L’Homme, n² 212, p. 109-138.

HARSGOR M. (1975), « L'essor des bâtards nobles au XVe siècle », Revue historique, $\mathrm{n}^{\circ}$ 514, p. 319-354.

JACQUART D., BuRnETt C. (dir.) (2005), Scientia in margine : études sur les Marginalia dans les manuscrits scientifiques du Moyen Âge à la Renaissance, Genève, Droz.

Lamont M., MolnÁr V. (2002), "The Study of Boundaries in the Social Sciences », Annual Review of Sociology, $\mathrm{n}^{\circ}$ 28, p. 167-195.

La Mure J.-M. De (1982 [1675]), Histoire des ducs de Bourbon et des comtes de Forez, Roanne, Horvath.

Le Dantec-Mowry H., Rossignol M.-J., Renault M., Vermeren P. (dir.) (2018), Histoire en marges. Les périphéries de l'histoire globale, Tours, Presses Universitaires FrançoisRabelais. 
Lefebvre-Teillard A. (1976), « L'enfant naturel dans l'ancien droit français », Recueil de la société Jean Bodin, vol. 36, n² 2, Bruxelles, Édition de la librairie encyclopédique, p. 251-269.

Lefebvre-Teillard A. (2008), Autour de l'enfant: du droit canonique et romain médiéval au Code Civil de 1804, Leiden, Brill.

Lett D. (2017), « De l'usage de la famille dans l'Histoire médiévale », Ménestrel. En ligne, consulté le 24 avril 2019. URL : http://www.menestrel.fr/?-famille-.

LouTsch J.-C. (1988), " Le franc-quartier, pièce honorable ou brisure ? », Brisures, augmentations et changements d'armoiries. Actes du Ve colloque d'Héraldique de Spolète, Bruxelles, Académie Internationale d'Héraldique, p. 139-162.

Mathevot C. (2011), " Le château de Bouthéon et les Bourbon (1462-1519) ", Forez et Bourbon. Les ducs de Bourbon maîtres du Forez aux XIV ${ }^{e}-X V^{e}$ siècles. Actes du colloque de Montbrison du 23 octobre 2010, Montbrison, La Diana, p. 117-139.

MatTÉoni O. (1998), Servir le prince. Les officiers des ducs de Bourbon à la fin du Moyen Âge (1356-1523). Étude d'une société politique, Paris, Publications de la Sorbonne.

MATTÉONI O. (2009), « Société contractuelle, pouvoir princier et domination territoriale : les alliances du duc Jean I ${ }^{\text {er }}$ de Bourbon avec la noblesse d'Auvergne (1413-1415) » in M. Gentile, P. Savy (dir.), Noblesse et États princiers en Italie et en France au XV $\mathrm{XV}^{e}$ siècle, Rome, Centre Jean Bérard, p. 287-334.

McDougall S. (2017), Royal Bastards. The Birth of Illegitimacy, 800-1230, Oxford, Oxford University Press.

Morsel J. (2017), Noblesse, parenté et reproduction sociale à la fin du Moyen Âge, Paris, Picard.

Pinoteau H. (1979), Héraldique capétienne, Paris, P. de la Perrière.

Prost B. (2004), « Marge et dynamique territoriale », Géocarrefour, vol. 79, n 2, p. 175-182.

Roumy F. (2000), « De la théologie au droit: naissance médiévale du concept de filiation », in R. Helmotz, P. Mikat, J. MülleR, M. Stolleis (dir.), Grundlagen des Rechts. Festschrift für Peter Landau zum 65 Geburstag, Paderborn, Ferdinand Schönigh (Rechts-und Staatswissenschatliche Veröffentlichungen des Görres-Gesellschaft, 91), p. 412-438.

Roumy F. (2009), « La contribution du droit canonique médiéval à l'élaboration d'une typologie des filiations dans les droits européens contemporains », in O. CoNDORELLI, F. Roumy, M. Schmoeckel (dir.), Der Einfluss der Kanonistik auf die europäische Rechtskultur 1. Zivil und Zivilprozessrecht, Cologne, Böhlau, p. 265-285.

Schmitt J.-C. (1988 [1978]), « L'histoire des marginaux », in J. Le Goff (dir.), La nouvelle histoire, Paris, Ed. Complexes, p. 277-305. 
Schnerb B. (2015), « Des bâtards nobles au service du prince : l'exemple de la cour de Bourgogne (fin XIVe-début XVe siècle), in É. Bousmar, A. Marchandisse, C. Masson, B. SCHNerb (dir.), La bâtardise et l'exercice du pouvoir en Europe du XIIIe au début du XVIe siècle, Lilles, Revue du Nord, Hors-Série n 31, coll. « Histoire », p. 91-112.

StASZAK J.-F. (2017), Frontières en tous genres. Cloisonnement spatial et constructions identitaires, Rennes, PUR.

Steinberg S. (2012), " "Au défaut des mâles”. Genre, succession féodale et idéologie nobiliaire (France, XVI ${ }^{\mathrm{e}}$-XVII ${ }^{\mathrm{e}}$ siècles) », Annales HSS, nº 3, p. 679-713.

Steinberg S. (2016), Une tache au front. La bâtardise aux XVI et XVII e siècles, Paris, Albin Michel.

WeBER F. (2005), Le sang, le nom, le quotidien : une sociologie de la parenté pratique, La Courneuve, Aux lieux d'être. 